\title{
Cyclophilin A protects HIV-1 from restriction by human TRIM5a
}

Kyusik Kim ${ }^{1}$, Ann Dauphin ${ }^{1}$, Sevnur Komurlu², Leonid Yurkovetskiy ${ }^{1}$, William E. Diehl ${ }^{1}$, Sean M. McCauley ${ }^{1}$, Claudia Carbone ${ }^{1}$, Caterina Strambio-De-Castillia ${ }^{1}$, Edward M. Campbell ${ }^{2,3}$, and Jeremy Luban ${ }^{1,4 *}$

${ }^{1}$ Program in Molecular Medicine, University of Massachusetts Medical School, Worcester, MA 01605, USA

${ }^{2}$ Department of Microbiology and Immunology, Stritch School of Medicine, Loyola University Chicago, Maywood, IL 60153, USA

${ }^{3}$ Infectious Disease and Immunology Institute, Stritch School of Medicine, Loyola University Chicago, Maywood, IL 60153, USA

${ }^{4}$ Department of Biochemistry and Molecular Pharmacology, University of Massachusetts Medical School, Worcester, MA 01605, USA

${ }^{*}$ Correspondence: Jeremy.luban@umassmed.edu (J.L.) 
1 The capsid (CA) protein lattice of HIV-1 and other retroviruses encases viral

2 genomic RNA and regulates steps that are essential to retroviral invasion of

3 target cells, including reverse transcription, nuclear trafficking, and integration of

4 viral cDNA into host chromosomal DNA 1 . Cyclophilin A (CypA), the first cellular

5 protein reported to bind HIV-1 $\mathrm{CA}^{2}$, has interacted with invading lentiviruses

6 related to HIV-1 for millions of years $^{3-7}$. Disruption of the CA-CypA interaction

7 decreases HIV-1 infectivity in human cells ${ }^{8-12}$, but stimulates infectivity in non-

8 human primate cells ${ }^{13-15}$. Genetic and biochemical data suggest that CypA

9 interaction with CA protects HIV-1 from a restriction factor in human cells ${ }^{16-20}$.

10 Discovery of the CA-specific restriction factor TRIM5 $\alpha^{21}$, and of TRIM5-CypA

11 fusion genes that were independently generated at least four times in

12 phylogeny $4,5,15,22-25$, pointed to human TRIM5a as the CypA-sensitive restriction

13 factor. However, significant HIV-1 restriction by human TRIM5 $\alpha^{21}$, let alone

14 inhibition of such activity by $\mathrm{CypA}^{26}$, has not been detected. Here, exploiting

15 reverse genetic tools optimized for primary human $\mathrm{CD4}^{+} \mathrm{T}$ cells, macrophages,

16 and dendritic cells, we demonstrate that disruption of the CA-CypA interaction

17 renders HIV-1 susceptible to restriction by human TRIM5a, with the block

18 occurring before reverse transcription. Identical findings were obtained with

19 single-cycle vectors or with replication-competent HIV-1, including sexually-

20 transmitted clones from sub-Saharan Africa. Endogenous TRIM5a was observed

21 to associate with virion cores as they entered the macrophage cytoplasm, but

22 only when the CA-CypA interaction was disrupted. These experiments resolve the

23 long-standing mystery of the role of CypA in HIV-1 replication by demonstrating

24 that this ubiquitous cellular protein shields HIV-1 from previously inapparent, but

25 potent inhibition, imposed by human TRIM5a. Hopefully this reinvigorates

26 development of CypA-inhibitors for treatment of HIV-1 and other CypA-dependent

27 pathogens ${ }^{27-30}$. 
To assess the role of TRIM5 $\alpha$ and CypA in the primary human blood cell types that serve as targets for HIV-1 infection in vivo, lentiviral vectors were optimized for titer and knockdown efficiency in these cells ${ }^{26,31-34}$. Human macrophages, dendritic cells, and $\mathrm{CD}^{+} \mathrm{T}$ cells were transduced with lentivectors bearing a puromycin resistance cassette and shRNAs targeting either TRIM5 or luciferase (Luc) as a control. After three days of selection in puromycin, knockdown was confirmed by RT-qPCR for TRIM5 mRNA, and by rescue of N-MLV restriction (Extended Data Fig. 2a-c), as done previously ${ }^{26,31}$. TRIM5 and Luc control knockdown cells were then challenged with single-cycle, VSV G-pseudotyped, HIV-1-GFP reporter vectors. Three days later, the percentage of $\mathrm{GFP}^{+}$cells was assessed by flow cytometry as a measure of infectivity (Extended Data Fig. 1 shows the gating strategy).

As compared with Luc control knockdown, TRIM5 knockdown had minimal effect on HIV-1 transduction efficiency in macrophages, dendritic cells, or CD4 ${ }^{+} \mathrm{T}$ cells (Fig. 1a-d; Extended Data Fig. 3a). Infectivity of HIV-1 CA-P90A, a mutant that disrupts CypA binding 8,9 , was significantly attenuated in control knockdown cells generated with all three cell types (Fig. 1a-d). The effect was evident in cells from all blood donors tested (at least three blood donors per condition) and over a 100-fold range in challenge vector titer (Extended Data Fig. 3a). TRIM5 knockdown in macrophages, dendritic cells, or $\mathrm{CD}^{+} \mathrm{T}$ cells increased CA-P90A infectivity (Fig. 1a-d; Extended Data Fig 3a). Results were the same whether challenge was with a three-plasmid vector system based on the clade B HIV-1 NL4-3 lab strain ${ }^{33,35}$ (Fig. 1a-C), or a two plasmid vector system based on the clade C HIV-1zm249M transmission-founder strain from Zambia ${ }^{33,36}$ (Fig. 1d).

Given previous reports that CypA and TRIM5 act independently to regulate HIV-1 transduction of immortalized cell lines ${ }^{26}$, the rescue of CA-P90A infectivity by TRIM5 knockdown in primary human blood cells was surprising. Complementary

27 CypA interaction. For pharmacologic disruption, cells were incubated in media containing small molecules that compete with CA for binding to CypA $2,8-10,37$. As compared with DMSO solvent alone, cyclosporine A (CsA) reduced HIV-1 transduction

30 efficiency in control Luc knockdown macrophages (Fig. 1e). In contrast, cyclosporine $\mathrm{H}$, 31 an analogue with 1,000-fold lower affinity for CypA ${ }^{38}$, caused slight increase in HIV-1 
1 infection (Extended Data Fig. 3b). Either of two non-immunosuppressive CypA inhibitors 2 derived from sanglifehrin $\mathrm{A}^{37}$, decreased HIV-1 transduction efficiency in primary CD4+ 3 T cells (Fig. 1f; Extended Data Fig. 3c-e). TRIM5 knockdown reversed the HIV-1 4 inhibition in macrophages caused by CsA (Fig. 1e), or in $\mathrm{CD}^{+} \mathrm{T}$ cells caused by the 5 sanglifehrin A-derivatives (Fig. 1f; Extended Data Fig. 3c-e).

To disrupt the CA-CypA interaction using a genetic approach, macrophages were transduced with two vectors. The first vector conferred puromycin resistance and 8 expressed shRNAs targeting either TRIM5 or Luc. The second vector conferred blasticidin resistance and expressed shRNAs targeting either CypA or Luc. After simultaneous transduction with pairs of these vectors, macrophages were selected for three days in both puromycin and blasticidin and then challenged with single-cycle, VSV G-pseudotyped, HIV-1-GFP reporter vector bearing wild-type CA. As compared with the control Luc knockdown, CypA knockdown reduced CypA protein levels $\sim 70 \%$ (Extended Data Fig. 2d). Like CA-P90A and the small molecule inhibitors, CypA knockdown decreased transduction efficiency (Fig. 1g). This effect was rescued by simultaneous knockdown of TRIM5 (Fig. 1e) without restoring CypA protein levels (Extended Data Fig. 2d).

Though the shRNA that targets TRIM5 is distinguished from the next most similar sequence in the human genome (GRCh38) by multiple mismatches, off-target effects are theoretically possible. To test whether TRIM5 is sufficient to explain the HIV-1 restriction activity associated with CA-CypA disruption, a vector was designed based on the ubiquitin fusion technique ${ }^{39}$, that expresses a tripartite fusion of puromycin $\mathrm{N}$-acetyl transferase (PAC), ubiquitin ${ }^{\mathrm{K} 48 \mathrm{R}}$, and coding sequence for a protein of interest, in addition to an shRNA (Fig. 2a). Four variants of the plasmid were engineered in which the shRNA targeted either TRIM5 or Luc, with or without a TRIM5 $\alpha$ coding sequence that bears mismatches in the shRNA target sequence (Fig. 2a).

Macrophages (Fig. 2b) and CD4 ${ }^{+} \mathrm{T}$ cells (Fig. 2c) were transduced with each of the four variants of the shRNA tripartite fusion vector and selected for three days with puromycin. Cells were then challenged with the three-plasmid HIV-1-GFP reporter 30 vector used in Fig. 1a, and assessed by flow cytometry for percent GFP positive cells 31 three days later. As in Fig. 1, the infectivity of vector bearing wild-type CA was minimally 
1 affected by TRIM5 knockdown, or by TRIM5 overexpression (Fig. 2b, c). As compared

2 to the wild-type, the infectivity of vector bearing CA-P90A was decreased (Fig. 2b, c),

3 and the infectivity of this mutant was rescued by TRIM5 shRNA (Fig. 2b, c). In the

4 presence of the shRNA targeting TRIM5, delivery of TRIM5a coding sequence bearing

5 shRNA target-site mismatches restored restriction activity to the control level (Fig. 2b, c). These results demonstrate that, in primary human blood cells, human TRIM5a is sufficient to restrict HIV-1 transduction, but only when the CA-CypA interaction is 8 disrupted.

To determine at which step in the virus life cycle human TRIM5a inhibits HIV-1

11 transcription was assessed by qPCR. Macrophages and $\mathrm{CD}^{+} \mathrm{T}^{+}$cells were stably transduced and selected with vector expressing shRNAs targeting TRIM5 or Luc control (Fig $3 a, b)$, or with each of the four variants of the shRNA tripartite fusion vector (Fig. $3 c, d)$. Cells were then challenged with an HIV-1 reporter vector that has the 34 basepair loxP sequence in $\mathrm{U} 3$ to distinguish reporter vector transcripts from those of the shRNA lentivector ${ }^{40}$. DNA was collected $20 \mathrm{hrs}$ post-challenge and qPCR was performed using primers specific for full-length linear HIV-1 cDNA (late RT). In all experiments, reporter vector bearing the RT-D185K/D186L loss-of-function mutation ${ }^{40}$ was included as a control for background signal not due to nascent reverse transcription (Fig. 3).

In Luc control knockdown macrophages and $\mathrm{CD}^{+} \mathrm{T}$ cells, viral cDNA was reduced by CA-P90A, and this reduction was restored by TRIM5 knockdown (Fig. 3a, b). Viral cDNA was also reduced by CA-P90A in either cell type transduced with the control shRNA tripartite fusion vector (Fig. 3c, d). TRIM5 shRNA rescued the cDNA (Fig. 3a-d), and rescue of TRIM5a with the non-targetable coding sequence again decreased the CA-P90A cDNA (Fig. 3c, d). These results demonstrate that, when the CA-CypA interaction is disrupted, human TRIM5a blocks HIV-1 at an early step of viral infection, prior to completion of reverse transcription.

To determine if TRIM5a associates with HIV-1 CA in cells when the CA-CypA 30 interaction is disrupted, primary human macrophages were stably transduced with 31 TRIM5 shRNA or Luc shRNA and then challenged for 2 hrs with wild-type HIV-1 
1 reporter vector, in the presence or absence of CsA. Cells were fixed and subjected to 2 the proximity ligation assay (PLA) with antibodies specific for HIV-1 CA and 3 endogenous human TRIM5a. When cells were challenged with HIV-1 in the absence of 4 CsA, very few puncta were detected (Fig. 4a, b; Extended Data Fig. 4). Similarly, few 5 puncta were detected when cells were treated with CsA in the absence of HIV-1 challenge. In contrast, when cells were challenged with HIV-1 in the presence of CsA, multiple puncta were detected (Fig. 4a, b), an increase of at least 20-fold in the average 8 number of puncta per cell over the background (Fig. 4b; Extended Data Fig. 4). TRIM5 knockdown eliminated the puncta (Fig. 4a, b; Extended Data Fig. 4), indicating that the PLA signal was dependent upon TRIM5 expression. These results indicate that, in acutely infected primary human macrophages, endogenous human TRIM5a associates with HIV-1 CA when the CA-CypA interaction is disrupted.

The above experiments used exogenous, single-cycle HIV-1 vectors. The effect of CypA on HIV-1 restriction by human TRIM5a was therefore evaluated next using replication-competent HIV-1 in a context where the virus spreads cell-to-cell. Primary human macrophages were challenged with clade B HIV-1 bearing a macrophage-tropic env (HIV-1 $\mathrm{MAC}$ ) and replication was monitored for 14 days by measuring the accumulation of reverse transcriptase activity in the supernatant. As in the single cycle experiments (Fig. 1), TRIM5 knockdown itself had little effect on HIV-1 replication (Fig. 5a, b). Disruption of the CA-CypA interaction with CsA (Fig. 5a) or with shRNA targeting CypA (Fig. 5b) effectively suppressed viral spread in the culture, and, in both cases, replication kinetics was completely restored to the control level by shRNA targeting TRIM5 (Fig. 5a, b). Primary $\mathrm{CD}^{+} \mathrm{T}$ cells were then challenged with a clade $\mathrm{C}$ transmission/founder virus (HIV-12m249M). As observed in macrophages, TRIM5 knockdown alone had minimal effect on wild-type HIV-1 replication (Fig. 5c, d). No viral replication was detectable when the CA-CypA interaction was disrupted by the small 27 molecule GS-CypAi3 (Fig. 5c) or by the presence of CA-P90A in HIV-1 (Fig. 5d); in both cases shRNA targeting TRIM5 rescued replication kinetics to the level of controls (Fig. 5c, d).

The experiments presented here demonstrate that, in primary human blood cells,

31 HIV-1 exploits CypA to evade CA recognition by, and the antiviral activity of, 
1 endogenous TRIM5 $\alpha$. This answers the long-standing question of how CypA promotes

2 HIV-1 infection and clearly establishes that, in the absence of CypA, human TRIM5a

3 potently restricts HIV-1. Conservation of the lentiviral CA-CypA interaction across

4 millions of years of evolution is likely a result of selective pressure applied by TRIM5a

5 orthologues encoded by host species that are otherwise permissive for lentiviral

6 replication. Finally, the results here, in which primary human blood cells were

7 challenged by clones of HIV-1 that were isolated after human-to-human transmission,

8 indicate that, by rendering HIV-1 susceptible to the potent antiviral activity of TRIM5a,

9 non-immunosuppressive CypA inhibitors have potential to make an important

10 contribution to anti-HIV-1 drug cocktails. 


\section{Methods}

Plasmids

4 All plasmids used here are described in Supplementary Table 1, and are available, 5 along with full sequences, at https://www.addgene.org/Jeremy Luban/.

\section{Human blood}

8 Leukopaks were obtained from anonymous, healthy, blood donors (New York Biologics, Southhampton, NY). These experiments were reviewed by the University of Massachusetts Medical School Institutional Review Board, and declared non-human subjects research, according to $\mathrm{NIH}$ guidelines (http://grants.nih.gov/grants/policy/hs/faqs_aps_definitions.htm).

\section{Cell culture}

All cells were cultured in humidified, $5 \% \mathrm{CO}_{2}$ incubators at $37^{\circ} \mathrm{C}$. HEK293 cells (ATCC) were cultured in DMEM supplemented with 10\% heat-inactivated FBS, $1 \mathrm{mM}$ sodium pyruvate, $20 \mathrm{mM}$ GlutaMAX ${ }^{\mathrm{TM}}{ }_{-}$, $1 \times$ MEM non-essential amino acids, and $25 \mathrm{mM}$ HEPES, pH 7.2 (DMEM-FBS complete). PBMCs were isolated from leukopaks by gradient centrifugation on Lymphoprep (Axis-Shield PoC As, Oslo, Norway, catalogue \#AXS-1114546). To generate dendritic cells (DCs) or macrophages, CD14

21 mononuclear cells were enriched by positive selection using anti-CD14 antibody 22 microbeads (Miltenyi, San Diego, CA, catalogue \#130-050-201). Enriched CD14 ${ }^{+}$cells 23 were plated in RPMI-1640, supplemented with $5 \%$ heat-inactivated human $\mathrm{AB}^{+}$serum 24 (Omega Scientific, Tarzana, CA), $1 \mathrm{mM}$ sodium pyruvate, $20 \mathrm{mM}$ GlutaMAX ${ }^{\mathrm{TM}}$-I, $1 \times$ 25 MEM non-essential amino acids, and 25 mM HEPES pH 7.2 (RPMI-HS complete), at a 26 density of $10^{6}$ cells $/ \mathrm{mL}$ for macrophages or $2 \times 10^{6}$ cells $/ \mathrm{mL}$ for DCs. To differentiate $27 \mathrm{CD} 14^{+}$cells into macrophages, 1:100 GM-CSF-conditioned media was added. To 28 differentiate $\mathrm{CD} 14^{+}$cells into DCs, 1:100 cytokine-conditioned media containing human 29 GM-CSF and human IL-4 was added. GM-CSF and IL-4 were produced from HEK293 30 cells transduced with pAIP-hGMCSF-co (Addgene \#74168) or pAIP-hIL4-co (Addgene $31 \# 74169)$, as previously described ${ }^{31,33}$. $\mathrm{CD}^{+}{ }^{+} \mathrm{T}$ cells were isolated from CD14-depleted 
PBMCs using anti-CD4 antibody microbeads (Miltenyi, catalogue \#130-045-101);

2 enrichment was typically $>90 \%$, as assessed by measuring the percentage of

$3 \mathrm{CD}^{+} / \mathrm{CD}^{+}$cells via flow cytometry with FITC-anti-CD3 (Biolegend, San Diego, CA,

4 catalogue \#317306) and APC-anti-CD4 (Biolegend, catalogue \#317416). The cells were

5 cultured in RPMI-1640 supplemented with 10\% heat-inactivated FBS, 1 mM sodium

6 pyruvate, $20 \mathrm{mM}$ GlutaMAX ${ }^{\mathrm{TM}}-\mathrm{I}$, 1× MEM non-essential amino acids, and $25 \mathrm{mM}$

7 HEPES pH 7.2 (RPMI-FBS complete) with 50 U/mL hIL-2 (NIH AIDS Reagent Program,

8 catalogue \#136).

\section{Virus production}

$1124 \mathrm{hrs}$ prior to transfection, $6 \times 10^{5}$ HEK293 cells were plated per well in 6-well plates.

12 All transfections used $2.49 \mu \mathrm{g}$ plasmid DNA with $6.25 \mu \mathrm{L}$ TransIT LT1 transfection

13 reagent (Mirus, Madison, WI), in $250 \mu \mathrm{L}$ Opti-MEM (Gibco). $2.49 \mu \mathrm{g}$ of replication-

14 competent HIV-1 provirus DNA was transfected. For two-part, single-cycle vector, 2.18

$15 \mu \mathrm{g}$ of env-defective HIV-1 provirus was co-transfected with $0.31 \mu \mathrm{g}$ pMD2.G VSV G plasmid. Three-part, single-cycle vectors were produced by co-transfecting $1.25 \mu \mathrm{g}$ minimal lentivector genome plasmid (either pALPS-GFP, pWPTS-GFP, pLXIN-GFP, pAPM-CoE-D4-miR30, pABM-CoE-D4-miR30, or pPU-ORF-miR30), $0.93 \mu \mathrm{g}$ gag-pol plasmid (either psPAX2, p8.9 N $\Delta$ SB, pCIG3-N or pCIG3-B), and 0.31 $\mu \mathrm{g}$ pMD2.G VSV G plasmid. Vpx-containing SIV-VLPs were produced by transfection of $2.18 \mu \mathrm{g}$ pSIV3+ and $0.31 \mu \mathrm{g}$ pMD2.G plasmid. $16 \mathrm{hrs}$ post-transfection, culture media was changed to the media specific for the cells to be transduced. Viral supernatant was harvested at 72 hrs, passed through a $0.45 \mu \mathrm{m}$ filter, and stored at $-80^{\circ} \mathrm{C}$.

\section{Exogenous reverse transcriptase assay}

$265 \mu \mathrm{L}$ transfection supernatant was mixed with $5 \mu \mathrm{L} 0.25 \%$ Triton $\mathrm{X}-100,50 \mathrm{mM} \mathrm{KCl}, 100$

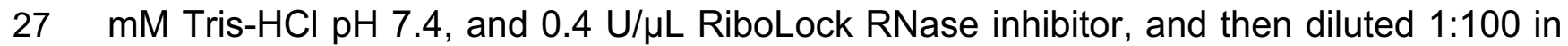
$285 \mathrm{mM}\left(\mathrm{NH}_{4}\right)_{2} \mathrm{SO}_{4}, 20 \mathrm{mM} \mathrm{KCl}$, and $20 \mathrm{mM}$ Tris- $\mathrm{HCl} \mathrm{pH} \mathrm{8.3.} 10 \mu \mathrm{L}$ of this was then added to a single-step, RT-PCR assay with $35 \mathrm{nM}$ MS2 RNA (IDT) as template, $500 \mathrm{nM}$

30 of each primer (5'-TCCTGCTCAACTTCCTGTCGAG-3' and 5'31 CACAGGTCAAACCTCCTAGGAATG-3'), and $0.1 \mu \mathrm{L}$ hot-start Taq DNA polymerase 
1 (Promega, Madison, $\mathrm{WI}$ ) in $20 \mathrm{mM}$ Tris- $\mathrm{Cl} \mathrm{pH} \mathrm{8.3,} 5 \mathrm{mM}\left(\mathrm{NH}_{4}\right)_{2} \mathrm{SO}_{4}, 20 \mathrm{mM} \mathrm{KCl}, 5 \mathrm{mM}$

$2 \mathrm{MgCl}_{2}, 0.1 \mathrm{mg} / \mathrm{ml} \mathrm{BSA}, 1 / 20,000$ SYBR Green I (Invitrogen), and $200 \mu \mathrm{M}$ dNTPs in total

$320 \mu \mathrm{L}$ reaction. The RT-PCR reaction was carried out in a Biorad CFX96 real-time PCR

4 detection system with the following parameters: $42^{\circ} \mathrm{C}$ for $20 \mathrm{~min}, 95^{\circ} \mathrm{C}$ for $2 \mathrm{~min}$, and 40

5 cycles $\left[95^{\circ} \mathrm{C}\right.$ for $5 \mathrm{sec}, 60^{\circ} \mathrm{C}$ for $5 \mathrm{sec}, 72^{\circ} \mathrm{C}$ for $15 \mathrm{sec}$, and acquisition at $80^{\circ} \mathrm{C}$ for 5 $6 \mathrm{sec}]$.

\section{Transduction with lentiviral knockdown vectors}

9 For DCs, $2 \times 10^{6} \mathrm{CD} 14^{+}$monocytes $/ \mathrm{mL}$ were transduced with 1:4 volume of SIV-VLPs and 1:4 volume of knockdown lentivector. For macrophages, $10^{6} \mathrm{CD} 14^{+}$monocytes $/ \mathrm{mL}$ were transduced with 1:8 volume of SIV-VLPs and 1:8 volume of knockdown lentivector.

12 The Vpx-containing SIV-VLPs were added to these cultures to overcome a SAMHD1 block to lentiviral transduction ${ }^{41,42}$. Transduced cells were selected with $3 \mu \mathrm{g} / \mathrm{mL}$ puromycin (InvivoGen, San Diego, CA, catalogue \#ant-pr-1), $10 \mu \mathrm{g} / \mathrm{mL}$ blasticidin (InvivoGen, catalogue \#ant-bl-1), or both, for 3 days, starting 3 days post-transduction.

Following isolation with magnetic beads, human $\mathrm{CD} 4^{+} \mathrm{T}$ cells were cultured at 2 to $3 \times 10^{6}$ cells $/ \mathrm{mL}$ in RPMI-FBS complete, supplemented with $50 \mathrm{U} / \mathrm{mL} \mathrm{hlL}-2$, and stimulated with $5 \mu \mathrm{g} / \mathrm{mL}$ PHA-P (Sigma-Aldrich, catalogue \#L-1668). Alternatively, CD4 ${ }^{+}$ T cells at $10^{6}$ cells $/ \mathrm{mL}$ were stimulated with $25 \mu \mathrm{L} / \mathrm{mL}$ ImmunoCult ${ }^{\mathrm{TM}}$ Human CD3/CD28 T Cell Activator (STEMCELL Technologies, Vancouver, Canada, catalogue \#10991). At day 3 post-stimulation, T cells were replated at 2 to $3 \times 10^{6}$ cells $/ \mathrm{mL}$ in RPMI-FBS complete, with $50 \mathrm{U} / \mathrm{mL}$ hIL-2. Cells were transduced with $10^{8} \mathrm{RT}$ units of viral vector per $10^{6}$ cells for 3 days, followed by selection with $2 \mu \mathrm{g} / \mathrm{mL}$ puromycin. After selection for 3 days, cells were re-stimulated with PHA-P, or with ImmunoCult ${ }^{\mathrm{TM}}$ Human

25 CD3/CD28 T Cell Activator, for 3 days. The stimulated cells were then replated at 2 to 3 $26 \times 10^{6}$ cells $/ \mathrm{mL}(\mathrm{PHA})$ or at $10^{6} \mathrm{cells} / \mathrm{mL}$ (CD3/CD28) in RPMI-FBS complete with 50

$27 \mathrm{U} / \mathrm{mL} \mathrm{hIL-2}$, and challenged with lentiviral vectors for assessment of single-cycle 28 infectivity or spreading infection. Fresh media containing hIL-2 was replenished every 2 to 3 days. 
1 For human DCs, $2.5 \times 10^{5}$ cells were seeded per well, in a 48-well plate, on the day of

2 virus challenge. Media containing VSV G-pseudotyped lentiviral vector expressing GFP

3 (HIV-1-GFP) was added to challenge cells in a total volume of $250 \mu \mathrm{L}$. For human

4 macrophages, $2.5 \times 10^{5}$ cells were seeded per well in a 24 -well plate, and challenged

5 with HIV-1-GFP in a total volume of $500 \mu \mathrm{L}$. 1:50 volume of SIV VLPs was also added

6 to the medium during virus challenge of DCs or macrophages. To challenge human

$7 \mathrm{CD}^{+}$cells activated with PHA, $5 \times 10^{5}$ cells were plated per well in 96-well plate at 3

8 days after the second PHA stimulation. For $\mathrm{CD}^{+}$cells stimulated with CD3/CD28

9 activator, $2 \times 10^{5}$ cells were plated in each well of a 96-well plate, 3 days after

10 secondary stimulation. Cells were then challenged with GFP reporter viruses in a total

11 volume of $200 \mu \mathrm{L}$. For all three cell types, 4 dilutions of viral stocks, from $10^{5}$ to $10^{8} \mathrm{RT}$

12 units $/ \mathrm{mL}$, were used to challenge cells. Where indicated, cells were pre-treated with 8

$13 \mu \mathrm{M}$ cyclosporine $\mathrm{A}(\mathrm{CsA}), 8 \mu \mathrm{M}$ cyclosporine $\mathrm{H}(\mathrm{CsH})$, or $2.5 \mu \mathrm{M}$ of non-

14 immunosuppressive CypA inhibitors from Gilead (GS-CypAi3 or GS-CypAi48) ${ }^{37}$, for 1 hr

15 prior to virus challenge. In experiments using $\mathrm{CsH}$ treatment, the media was replaced

16 after $16 \mathrm{hrs}$ treatment in order to avoid $\mathrm{CsH}$ toxicity ${ }^{43}$. At $48 \mathrm{hrs}$ post-challenge with 2-

17 part HIV-1 vectors, or at 72 hrs post-challenge with 3-part lentiviral vectors, cells were

18 harvested for flow cytometric analysis, by pipetting (CD4 ${ }^{+}$T cells) or scraping (DCs and

19 macrophages). Cells were pelleted at $500 \times \mathrm{g}$ for $5 \mathrm{~min}$, and fixed in a 1:4 dilution of BD

20 Cytofix Fixation Buffer with phosphate-buffered saline (PBS) without $\mathrm{Ca}^{2+}$ and $\mathrm{Mg}^{2+}$,

21 supplemented with $2 \%$ FBS and $0.1 \% \mathrm{NaN}_{3}$.

\section{Flow Cytometry}

24 Data was collected on an Accuri C6 (BD Biosciences, San Jose, CA) and plotted with

25 FlowJo software. Infectivity at each dilution, in each condition (CA mutant, CypA 26 inhibitor, or CypA knockdown) was compared to the infectivity of WT CA in the control 27 condition. Dilutions yielding infectivity greater than $30 \% \mathrm{GFP}^{+}$cells were excluded from 28 analysis on the assumption that these were out of the linear range, according to the 29 Poisson distribution.

31 Statistical analysis 
1 Experimental $\mathrm{n}$ values and information regarding the statistical tests can be found in the

2 figure legends. The data of infectivity assay using single-cycle viruses including at least

3 three independent donors were statistically analyzed by using two-tailed paired t-test

4 compared to the control condition or the indicated condition for each donor. The data of

5 PLA quantification were assessed for statistical significance using two-tailed unpaired t-

6 test to compare two conditions as indicated in Fig. 4. All statistical analyses were

7 performed using PRISM 7.0 (GraphPad Software, La Jolla, CA).

\section{Quantitative PCR for viral late reverse transcriptase product}

10 Total DNA was extracted from cells using DNeasy Blood \& Tissue Kit (Qiagen, Hilden,

11 Germany), following the manufacturer's instruction. Late RT products were detected

12 with TaqMan system using the primers pWPTS J1B fwd and pWPTS J2 rev with the late

13 RT probe (LRT-P) ${ }^{44}$. Mitochondrial DNA was used for normalization with the following 14 primer/probe set: MH533, MH534 and Mito probe ${ }^{45}$. The primer and probe sequences 15 are specified in Supplementary Table 3. The quantitative PCR was performed in $20 \mu \mathrm{L}$ 16 reaction mix containing 1× TaqMan Gene Expression Master Mix (Applied Biosystems), $17900 \mathrm{nM}$ each primer, $250 \mathrm{nM}$ TaqMan probe and 30 to $50 \mathrm{ng}$ template DNA. After an 18 initial incubation at $50{ }^{\circ} \mathrm{C}$ for $2 \mathrm{~min}$ and the second incubation at $95^{\circ} \mathrm{C}$ for $10 \mathrm{~min}, 45$ 19 cycles of amplification were carried out at $95^{\circ} \mathrm{C}$ for $15 \mathrm{sec}$ followed by 1 min and $30 \mathrm{sec}$ at $60^{\circ} \mathrm{C}$. Real-Time PCR reactions were run on a CFX96 ${ }^{\mathrm{TM}}$ thermal cycler (Bio-Rad).

22 qRT-PCR

23 Total RNA was isolated in TRIzol reagent followed by RNA purification with RNeasy 24 Plus Mini kit (Qiagen). First-strand cDNA was generated using SuperScript ${ }^{\text {TM }}$ VILO $^{\text {TM }}$ 25 Master Mix (Thermo Fisher) with random hexamers, in accordance with manufacturer's 26 instructions. Duplex qPCR was performed in $20 \mu \mathrm{L}$ reaction mix containing $1 \times$ TaqMan 27 Gene Expression Master Mix, 1× TaqMan Gene Expression Assay detecting TRIM5 28 (FAM dye-labeled, TaqMan probe ID \#Hs01552559_m1), 1× TaqMan Gene Expression 29 Assay targeting a housekeeping gene OAZ1 (VIC dye-labeled, primer-limited, TaqMan 30 probe ID \#Hs00427923_m1). Amplification was on a Biorad CFX96 real-time PCR 
1 detection system, using: $95^{\circ} \mathrm{C}$ for $10 \mathrm{~min}$, then 45 cycles of $95^{\circ} \mathrm{C}$ for $15 \mathrm{sec}$ and $60^{\circ} \mathrm{C}$

2 for $60 \mathrm{sec}$.

\section{Western blot}

5 Cells were lysed in Hypotonic Lysis Buffer: $20 \mathrm{mM}$ Tris-HCl, pH 7.5, $150 \mathrm{mM} \mathrm{NaCl}, 10$ mM EDTA, $0.5 \%$ NP-40, $0.1 \%$ Triton X-100, and cOmplete mini protease inhibitor (Sigma-Aldrich) for $20 \mathrm{~min}$ on ice. The lysates were mixed 1:1 with $2 \times$ Laemmli buffer

8 containing 1:20-diluted 2-mercaptoethanol, boiled for $10 \mathrm{~min}$, and centrifuged at 16,000 $9 \times \mathrm{g}$ for $5 \mathrm{~min}$ at $4^{\circ} \mathrm{C}$. Samples were run on $4-20 \%$ SDS-PAGE and transferred to nitrocellulose membranes. Membrane blocking, as well as antibody binding were in TBS

11 Odyssey Blocking Buffer (Li-Cor, Lincoln, NE). Primary antibodies used were rabbit anti-

12 СурА (1:10,000 dilution; Enzo Life Sciences, Farmingdale, NY, catalogue \#BML-SA296)

13 and mouse anti- $\beta$-actin (1:1,000 dilution; Abcam, Cambridge, UK, catalogue \#ab3280).

14 Goat anti-mouse-680 (Li-Cor, catalogue \#925-68070) and goat anti-rabbit-800 (Li-Cor, catalogue \#925-32211) as secondary antibodies were used at 1:10,000 dilutions. Blots were scanned on the Li-Cor Odyssey CLx.

\section{Proximity ligation assay (PLA)}

$2.5 \times 10^{5}$ macrophages were plated on $12 \mathrm{~mm}$ coverslips (Warner Instrument, Hamden, $\mathrm{CT}$, catalogue \#CS-12R15) in 24-well plates. Cells were spinoculated at 1,200 $\times \mathrm{g}$ using $6 \times 10^{8} \mathrm{RT}$ unit/mL of 3-part lentiviral vector (pALPS-GFP, p8.9N $\Delta S B$, and pMD2.G, generated as above) at $13^{\circ} \mathrm{C}$ for $2 \mathrm{hrs}$. Media was replaced with RPMI-HS complete containing $2 \mu \mathrm{M}$ MG132, and either DMSO or $5 \mu \mathrm{M}$ CsA, and cells were incubated at $37^{\circ}$ for $2 \mathrm{hrs}$. Coverslips were fixed with $3.7 \%$ formaldehyde (ThermoFisher) in $0.1 \mathrm{M}$ PIPES, $\mathrm{pH}$ 6.8, for $5 \mathrm{~min}$ at room temperature, and then incubated at room temperature for $1 \mathrm{hr}$ in PBS containing $0.1 \%$ saponin, 10\% donkey serum, $0.01 \%$ sodium azide,

27 mouse anti-TRIM5 $\alpha$ antibody (NIH AIDS Reagent Program, catalogue \#12271) at a 1:750 dilution and rabbit anti-HIV-1 CA (p24) antibody (Abcam, catalogue \#ab32352) at a 1:400 dilution. The samples were processed further using a Duolink® In Situ Red kit

30 (Sigma-Aldrich), following the instructions of the manufacturer. Then samples were 31 incubated with $10 \mu \mathrm{M}$ phalloidin (FITC) (Enzo Life Science) and $1 \mathrm{mg} / \mathrm{mL}$ Hoechst 
133342 (Invitrogen), in PBS containing 10\% donkey serum and $0.01 \%$ sodium azide for

$230 \mathrm{~min}$ at room temperature. Coverslips were mounted on slides and stored at $-20^{\circ} \mathrm{C}$.

3 Interaction was detected as fluorescent spots ( $\lambda_{\text {excitation/emission }} 598 / 634 \mathrm{~nm}$ ).

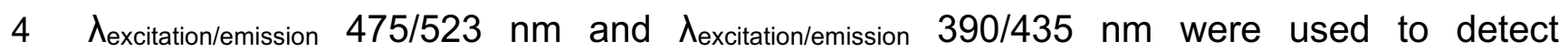

5 phalloidin and Hoechst, respectively. Z-stack images were collected with a DeltaVision

6 wide-field fluorescent microscope (Applied Precision, GE) and deconvolved with

7 SoftWoRx deconvolution software (Applied Precision, GE). All images were acquired

8 under identical acquisition conditions and analyzed by Imaris 8.3.1 (Bitplane). Three

9 dimensional representations were constructed by using the Easy 3D function (Imaris

10 8.3.1).

12 Challenge with replication-competent HIV-1

$135 \times 10^{5}$ macrophages per well in 12-well plates were challenged with $10^{8} \mathrm{RT}$ units of

14 HIV-1 for $2 \mathrm{hrs}$, in the presence of CsA or DMSO solvent, as indicated. Macrophage

15 experiments used NL4-3MAC; pNL4-3, in which env was replaced from the end of the

16 signal peptide to the env stop codon, with macrophage-tropic env from GenBank

17 \#U63632.1. 3 days after secondary stimulation with CD3/CD28, $10^{6} \mathrm{CD}^{+} \mathrm{T}$ cells per

18 well in 48-well plates were challenged with $2 \times 10^{7} \mathrm{RT}$ units of HIV-1 for $2 \mathrm{hrs}$, in the

19 presence of GS-CypAi3 or DMSO solvent, as indicated. CD4 ${ }^{+} \mathrm{T}$ cell experiments used

20 HIV-1 zм249м, a clade $C$ transmission-founder strain. After HIV-1 challenge, cells were

21 washed with fresh media and resuspended in $1 \mathrm{~mL}$ of RPMI-HS complete for

22 macrophages or RPMI-FBS complete containing $50 \mathrm{U} / \mathrm{mL} \mathrm{hIL-2} \mathrm{for} \mathrm{CD} 4^{+} \mathrm{T}$ cells. Where

23 indicated, culture media also contained CsA, GS-CypAi3, or DMSO solvent. Every 2-3

24 days, culture supernatant was harvested to measure RT activity. 


\section{References}

1. Yamashita, M. \& Engelman, A. N. Capsid-Dependent Host Factors in HIV-1 Infection. Trends Microbiol. 25, 741-755 (2017).

2. Luban, J., Bossolt, K. L., Franke, E. K., Kalpana, G. V. \& Goff, S. P. Human immunodeficiency virus type $1 \mathrm{Gag}$ protein binds to cyclophilins A and B. Cell 73, 1067-1078 (1993).

3. Goldstone, D. C., Yap, M. W., Robertson, L. E., Haire, L. F., Taylor, W. R., Katzourakis, A., Stoye, J. P. \& Taylor, I. A. Structural and functional analysis of prehistoric lentiviruses uncovers an ancient molecular interface. Cell Host Microbe 8, 248-259 (2010).

4. Malfavon-Borja, R., Wu, L. I., Emerman, M. \& Malik, H. S. Birth, decay, and reconstruction of an ancient TRIMCyp gene fusion in primate genomes. Proc. Natl. Acad. Sci. U. S. A. 110, E583-92 (2013).

5. Mu, D., Yang, H., Zhu, J.-W., Liu, F.-L., Tian, R.-R., Zheng, H.-Y., Han, J.-B., Shi, P. \& Zheng, Y.-T. Independent birth of a novel TRIMCyp in Tupaia belangeri with a divergent function from its paralog TRIM5. Mol. Biol. Evol. 31, 2985-2997 (2014).

6. Gilbert, C., Maxfield, D. G., Goodman, S. M. \& Feschotte, C. Parallel germline infiltration of a lentivirus in two Malagasy lemurs. PLoS Genet. 5, e1000425 (2009).

7. Katzourakis, A., Tristem, M., Pybus, O. G. \& Gifford, R. J. Discovery and analysis of the first endogenous lentivirus. Proc. Natl. Acad. Sci. U. S. A. 104, 6261-6265 (2007).

8. Braaten, D., Franke, E. K. \& Luban, J. Cyclophilin A is required for an early step in the life cycle of human immunodeficiency virus type 1 before the initiation of reverse transcription. J. Virol. 70, 3551-3560 (1996).

9. Franke, E. K., Yuan, H. E. \& Luban, J. Specific incorporation of cyclophilin A into HIV-1 virions. Nature 372, 359-362 (1994).

10. Thali, M., Bukovsky, A., Kondo, E., Rosenwirth, B., Walsh, C. T., Sodroski, J. \& Göttlinger, H. G. Functional association of cyclophilin A with HIV-1 virions. Nature 372, 363-365 (1994).

11. Braaten, D. \& Luban, J. Cyclophilin A regulates HIV-1 infectivity, as demonstrated by gene targeting in human T cells. EMBO J. (2001). at <http://emboj.embopress.org/content/20/6/1300.abstract>

12. Sokolskaja, E., Sayah, D. M. \& Luban, J. Target cell cyclophilin A modulates human immunodeficiency virus type 1 infectivity. J. Virol. 78, 12800-12808 (2004).

13. Towers, G. J., Hatziioannou, T., Cowan, S., Goff, S. P., Luban, J. \& Bieniasz, P. D. Cyclophilin A modulates the sensitivity of HIV-1 to host restriction factors. Nat. Med. 9, 1138-1143 (2003).

14. Berthoux, L., Sebastian, S., Sokolskaja, E. \& Luban, J. Cyclophilin A is required for TRIM5a-mediated resistance to HIV-1 in Old World monkey cells. Proc. Natl. Acad. Sci. U. S. A. 102, 14849-14853 (2005).

15. Sayah, D. M., Sokolskaja, E., Berthoux, L. \& Luban, J. Cyclophilin A retrotransposition into TRIM5 explains owl monkey resistance to HIV-1. Nature 430, 569-573 (2004).

16. Luban, J. Cyclophilin A, TRIM5, and resistance to human immunodeficiency virus type 1 infection. J. Virol. 81, 1054-1061 (2007). 
17. Sayah, D. M. \& Luban, J. Selection for loss of Ref1 activity in human cells releases human immunodeficiency virus type 1 from cyclophilin A dependence during infection. J. Virol. 78, 12066-12070 (2004).

18. Sebastian, S. \& Luban, J. TRIM5a selectively binds a restriction-sensitive retroviral capsid. Retrovirology 2, 1 (2005).

19. Sebastian, S., Sokolskaja, E. \& Luban, J. Arsenic counteracts human immunodeficiency virus type 1 restriction by various TRIM5 orthologues in a cell type-dependent manner. J. Virol. 80, 2051-2054 (2006).

20. Stremlau, M., Perron, M., Lee, M., Li, Y., Song, B., Javanbakht, H., Diaz-Griffero, F., Anderson, D. J., Sundquist, W. I. \& Sodroski, J. Specific recognition and accelerated uncoating of retroviral capsids by the TRIM5a restriction factor. Proc. Natl. Acad. Sci. U. S. A. 103, 5514-5519 (2006).

21. Stremlau, M., Owens, C. M., Perron, M. J., Kiessling, M., Autissier, P. \& Sodroski, J. The cytoplasmic body component TRIM5alpha restricts HIV-1 infection in Old World monkeys. Nature 427, 848-853 (2004).

22. Wilson, S. J., Webb, B. L. J., Ylinen, L. M. J., Verschoor, E., Heeney, J. L. \& Towers, G. J. Independent evolution of an antiviral TRIMCyp in rhesus macaques. Proc. Natl. Acad. Sci. U. S. A. 105, 3557-3562 (2008).

23. Virgen, C. A., Kratovac, Z., Bieniasz, P. D. \& Hatziioannou, T. Independent genesis of chimeric TRIM5-cyclophilin proteins in two primate species. Proc. Natl. Acad. Sci. U. S. A. 105, 3563-3568 (2008).

24. Newman, R. M., Hall, L., Kirmaier, A., Pozzi, L.-A., Pery, E., Farzan, M., O’Neil, S. P. \& Johnson, W. Evolution of a TRIM5-CypA splice isoform in old world monkeys. PLoS Pathog. 4, e1000003 (2008).

25. Brennan, G., Kozyrev, Y. \& Hu, S.-L. TRIMCyp expression in Old World primates Macaca nemestrina and Macaca fascicularis. Proc. Natl. Acad. Sci. U. S. A. 105, 3569-3574 (2008).

26. Sokolskaja, E., Berthoux, L. \& Luban, J. Cyclophilin A and TRIM5alpha independently regulate human immunodeficiency virus type 1 infectivity in human cells. J. Virol. 80, 2855-2862 (2006).

27. Frausto, S. D., Lee, E. \& Tang, H. Cyclophilins as modulators of viral replication. Viruses 5, 1684-1701 (2013).

28. Kaul, A., Stauffer, S., Berger, C., Pertel, T., Schmitt, J., Kallis, S., Lopez, M. Z., Lohmann, V., Luban, J. \& Bartenschlager, R. Essential role of cyclophilin A for hepatitis $C$ virus replication and virus production and possible link to polyprotein cleavage kinetics. PLoS Pathog. 5, e1000546 (2009).

29. Ma, C., Li, F., Musharrafieh, R. G. \& Wang, J. Discovery of cyclosporine A and its analogs as broad-spectrum anti-influenza drugs with a high in vitro genetic barrier of drug resistance. Antiviral Res. 133, 62-72 (2016).

30. Qing, M., Yang, F., Zhang, B., Zou, G., Robida, J. M., Yuan, Z., Tang, H. \& Shi, P.$Y$. Cyclosporine inhibits flavivirus replication through blocking the interaction between host cyclophilins and viral NS5 protein. Antimicrob. Agents Chemother. 53, 3226-3235 (2009).

31. Pertel, T., Hausmann, S., Morger, D., Züger, S., Guerra, J., Lascano, J., Reinhard, C., Santoni, F. A., Uchil, P. D., Chatel, L., Bisiaux, A., Albert, M. L., Strambio-DeCastillia, C., Mothes, W., Pizzato, M., Grütter, M. G. \& Luban, J. TRIM5 is an innate 
immune sensor for the retrovirus capsid lattice. Nature 472, 361-365 (2011).

32. Fellmann, C., Hoffmann, T., Sridhar, V., Hopfgartner, B., Muhar, M., Roth, M., Lai, D. Y., Barbosa, I. A. M., Kwon, J. S., Guan, Y., Sinha, N. \& Zuber, J. An optimized microRNA backbone for effective single-copy RNAi. Cell Rep. 5, 1704-1713 (2013).

33. McCauley, S. M., Kim, K., Nowosielska, A., Dauphin, A., Yurkovetskiy, L., Diehl, W. E. \& Luban, J. Intron-containing RNA from the HIV-1 provirus activates type I interferon and inflammatory cytokines. Nat. Commun. 9, 5305 (2018).

34. Yurkovetskiy, L., Guney, M. H., Kim, K., Goh, S. L., McCauley, S., Dauphin, A., Diehl, W. E. \& Luban, J. Primate immunodeficiency virus proteins Vpx and Vpr counteract transcriptional repression of proviruses by the HUSH complex. Nat Microbiol 3, 1354-1361 (2018).

35. Adachi, A., Gendelman, H. E., Koenig, S., Folks, T., Willey, R., Rabson, A. \& Martin, M. A. Production of acquired immunodeficiency syndrome-associated retrovirus in human and nonhuman cells transfected with an infectious molecular clone. J. Virol. 59, 284-291 (1986).

36. Salazar-Gonzalez, J. F., Salazar, M. G., Keele, B. F., Learn, G. H., Giorgi, E. E., Li, H., Decker, J. M., Wang, S., Baalwa, J., Kraus, M. H., Parrish, N. F., Shaw, K. S., Guffey, M. B., Bar, K. J., Davis, K. L., Ochsenbauer-Jambor, C., Kappes, J. C., Saag, M. S., Cohen, M. S., Mulenga, J., Derdeyn, C. A., Allen, S., Hunter, E., Markowitz, M., Hraber, P., Perelson, A. S., Bhattacharya, T., Haynes, B. F., Korber, B. T., Hahn, B. H. \& Shaw, G. M. Genetic identity, biological phenotype, and evolutionary pathways of transmitted/founder viruses in acute and early HIV-1 infection. J. Exp. Med. 206, 1273-1289 (2009).

37. Mackman, R. L., Steadman, V. A., Dean, D. K., Jansa, P., Poullennec, K. G., Appleby, T., Austin, C., Blakemore, C. A., Cai, R., Cannizzaro, C., Chin, G., Chiva, J.-Y. C., Dunbar, N. A., Fliri, H., Highton, A. J., Hui, H., Ji, M., Jin, H., Karki, K., Keats, A. J., Lazarides, L., Lee, Y.-J., Liclican, A., Mish, M., Murray, B., Pettit, S. B., Pyun, P., Sangi, M., Santos, R., Sanvoisin, J., Schmitz, U., Schrier, A., Siegel, D., Sperandio, D., Stepan, G., Tian, Y., Watt, G. M., Yang, H. \& Schultz, B. E. Discovery of a Potent and Orally Bioavailable Cyclophilin Inhibitor Derived from the Sanglifehrin Macrocycle. J. Med. Chem. 61, 9473-9499 (2018).

38. Husi, H. \& Zurini, M. G. Comparative binding studies of cyclophilins to cyclosporin A and derivatives by fluorescence measurements. Anal. Biochem. 222, 251-255 (1994).

39. Varshavsky, A. in Methods in Enzymology 399, 777-799 (Academic Press, 2005).

40. De laco, A. \& Luban, J. Inhibition of HIV-1 infection by TNPO3 depletion is determined by capsid and detectable after viral cDNA enters the nucleus. Retrovirology 8, 98 (2011).

41. Laguette, N., Sobhian, B., Casartelli, N., Ringeard, M., Chable-Bessia, C., Ségéral, E., Yatim, A., Emiliani, S., Schwartz, O. \& Benkirane, M. SAMHD1 is the dendriticand myeloid-cell-specific HIV-1 restriction factor counteracted by Vpx. Nature 474, 654-657 (2011).

42. Hrecka, K., Hao, C., Gierszewska, M., Swanson, S. K., Kesik-Brodacka, M., Srivastava, S., Florens, L., Washburn, M. P. \& Skowronski, J. Vpx relieves inhibition of HIV-1 infection of macrophages mediated by the SAMHD1 protein. 
Nature 474, 658-661 (2011).

43. Petrillo, C., Thorne, L. G., Unali, G., Schiroli, G., Giordano, A. M. S., Piras, F., Cuccovillo, I., Petit, S. J., Ahsan, F., Noursadeghi, M., Clare, S., Genovese, P., Gentner, B., Naldini, L., Towers, G. J. \& Kajaste-Rudnitski, A. Cyclosporine H Overcomes Innate Immune Restrictions to Improve Lentiviral Transduction and Gene Editing In Human Hematopoietic Stem Cells. Cell Stem Cell 23, 820-832.e9 (2018).

44. Reinhard, C., Bottinelli, D., Kim, B. \& Luban, J. Vpx rescue of HIV-1 from the antiviral state in mature dendritic cells is independent of the intracellular deoxynucleotide concentration. Retrovirology 11, 12 (2014).

45. Butler, S. L., Hansen, M. S. \& Bushman, F. D. A quantitative assay for HIV DNA integration in vivo. Nat. Med. 7, 631-634 (2001). 
Supplementary information is available in the online version of the paper.

Acknowledgements We thank Tomas Cihlar, Beatrice Hahn, Stephane Hausmann, Eric Hunter, Richard Mackman, Massimo Pizzato, and Stephen Yant for reagents. We are also grateful to anonymous blood donors who contributed leukocytes to this study. This work was supported by NIH Grants 5R01Al111809, 5DP1DA034990, and 1R01Al117839, to J.L.

Author Contributions K.K. and J.L. designed the experiments. K.K., A.D., S.K., L.Y., W.E.D., S.M.M., and C.C. conducted the experiments. C.S. and E.M.C provided advice and technical expertise. All authors analyzed the data. K.K. and J.L. wrote the manuscript.

Author information The authors declare no competing financial interests. Correspondence and requests for materials should be addressed to J.L. (jeremy.luban@umassmed.edu).

Data availability statement The plasmids described in Supplementary Table 1 are available at www.addgene.com. All data generated or analyzed during this study are presented in the paper or in the Supplementary Information file, as well as are available from the corresponding author upon reasonable request. 


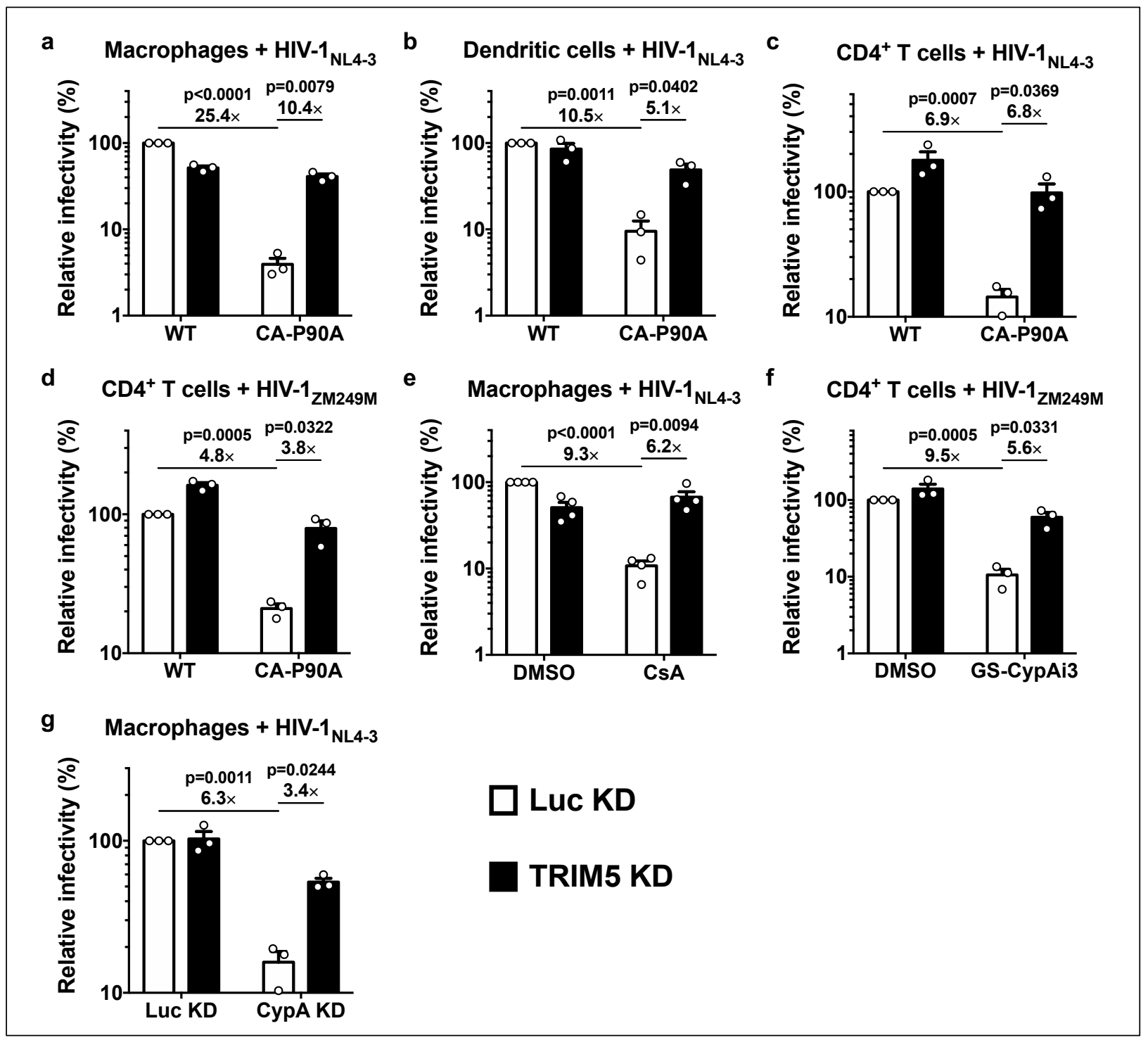

Fig. 1. Disruption of the CA-CypA interaction in primary human blood cells renders HIV-1 susceptible to restriction by TRIM5. a, Macrophages, b, dendritic cells, or $\mathbf{c}$ and $\mathbf{d}, \mathrm{CD}^{+} \mathrm{T}$ cells, were selected after transduction with a lentivirus expressing shRNA targeting TRIM5 or Luc control, and challenged with single-cycle,

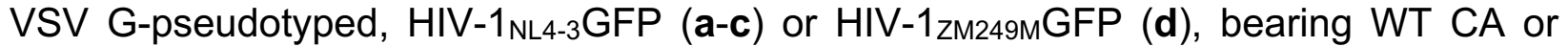
CA-P90A (mean \pm SEM, $\mathrm{n}=3$ donors for each). e, TRIM5 knockdown or Luc

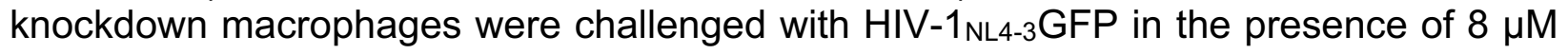
CsA or DMSO solvent (mean \pm SEM, $\mathrm{n}=4$ donors). $\mathrm{f}$, TRIM5 knockdown or Luc knockdown $\mathrm{CD}^{+} \mathrm{T}$ cells were challenged with HIV-1zм249мGFP in the presence of 2.5 $\mu \mathrm{M}$ GS-CypAi3 or DMSO solvent (mean \pm SEM, $n=3$ donors). g, Macrophages were transduced simultaneously with two vectors expressing shRNAs, as indicated, and selected with puromycin and blasticidin. Cells were then challenged with HIV-1 ${ }_{\text {NL4-3GFP }}$ (mean \pm SEM, $n=3$ donors). The percentage of $\mathrm{GFP}^{+}$cells was assessed by flow cytometry and normalized to WT in Luc control knockdown cells in all cases. Significance was determined by two-tailed, paired t-test. 


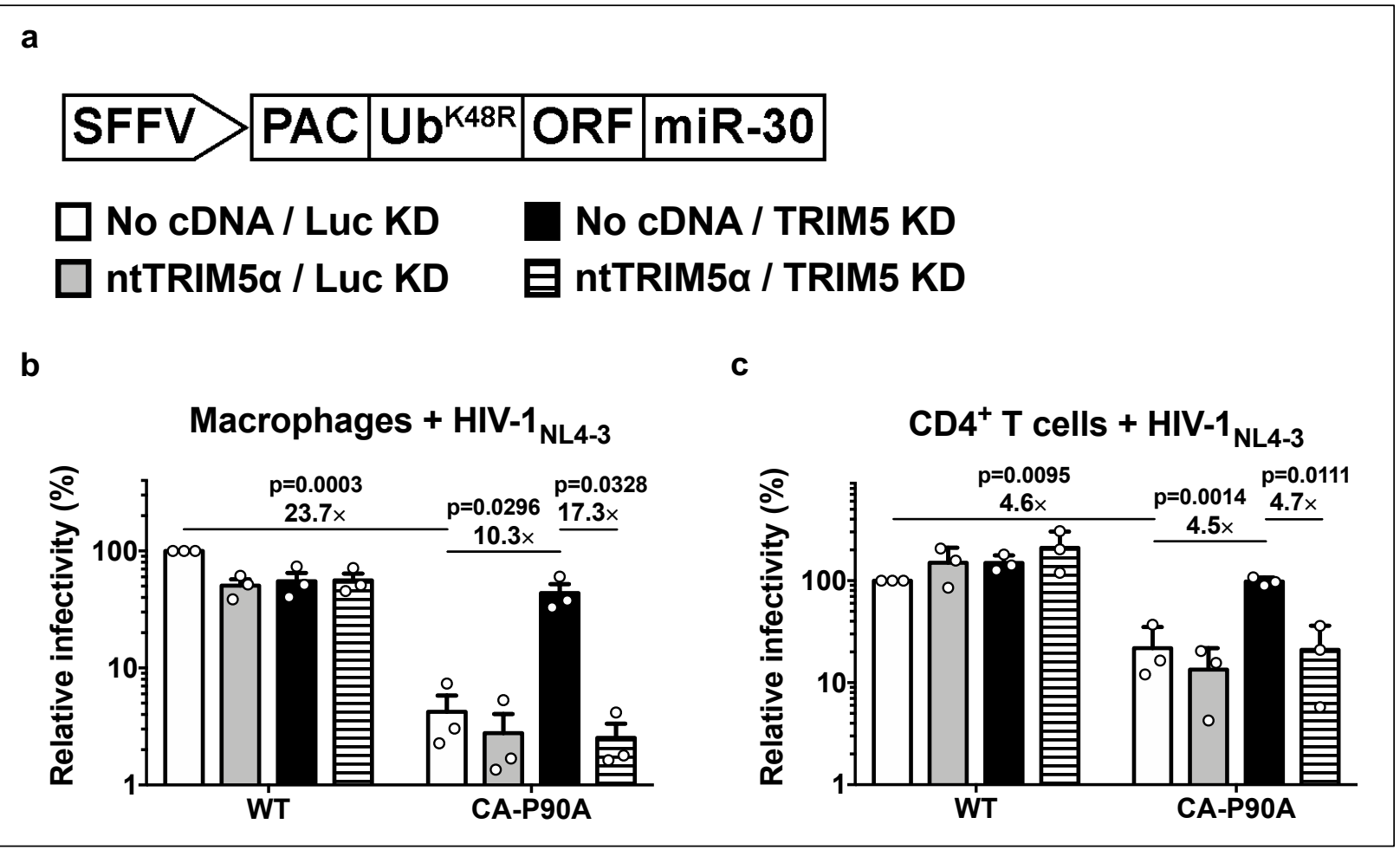

Fig. 2. Human TRIM5 $\alpha$ is sufficient to explain the HIV-1 inhibition that results from disruption of the CA-CypA interaction. a, Schematic representation of the all-in-one shRNA-rescue lentivector, in which the SFFV promoter expresses a tripartite fusion of puromycin $\mathrm{N}$-acetyl transferase (PAC), the K48R mutant of ubiquitin, and an open reading frame for a gene of interest (ORF), as well as a miR-30 based shRNA (miR-30). b and c, All-in-one lentivectors encoding the shRNAs and ORFs indicated in a were used to transduce macrophages (b) or $\mathrm{CD}^{+} \mathrm{T}$ cells (c). The percentage of GFPexpressing cells was measured by flow cytometry and normalized to the values for No cDNA/Luc KD cells challenged with WT CA; mean \pm SEM, $n=3$ donors for each. Significance was determined by two-tailed, paired t-test. 


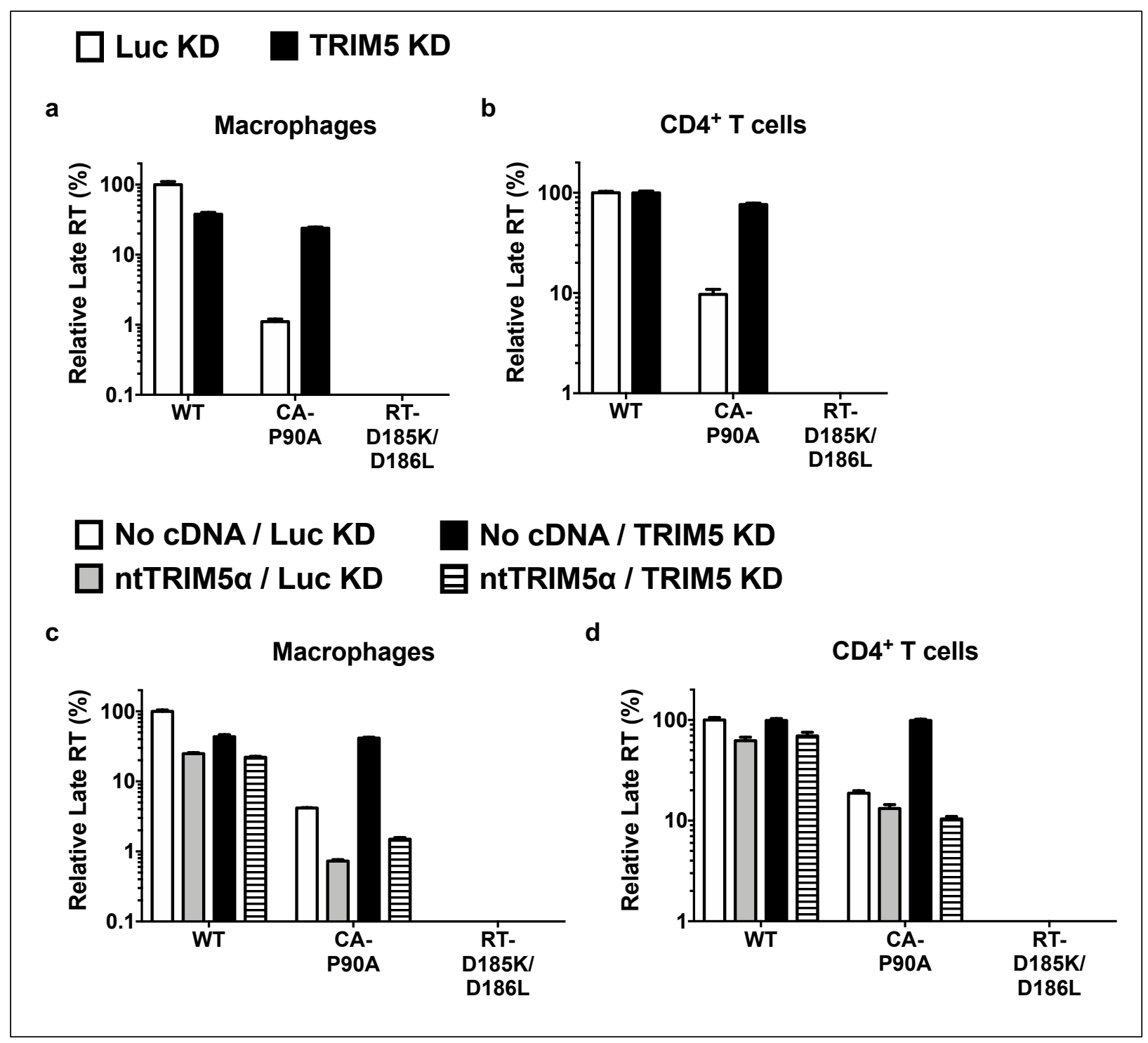

Fig. 3. CypA protects HIV-1 from restriction by human TRIM5a prior to completion of reverse transcription. a-d, TRIM5 knockdown or Luc knockdown macrophages (a) or $\mathrm{CD}^{+} \mathrm{T}$ cells (b), or macrophages (c) or $\mathrm{CD}^{+} \mathrm{T}$ cells (d) transduced with the all-inone shRNA-rescue lentivectors described in Fig. 2 were challenged with HIV-1 NL4-3 GFP containing WT CA or CA-P90A, as indicated. DNA was extracted $20 \mathrm{hrs}$ post-challenge and late products of reverse transcription (RT) were assessed by qPCR (mean of triplicates \pm SEM). RT-D185K/D186L mutant virus was used as a control for background. 
a

Donor 1

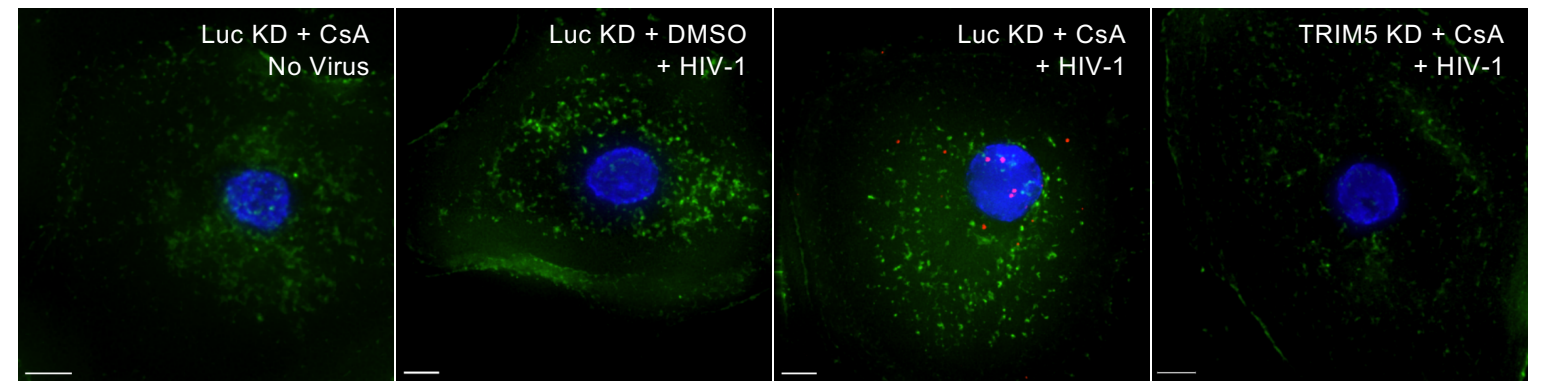

Donor 2
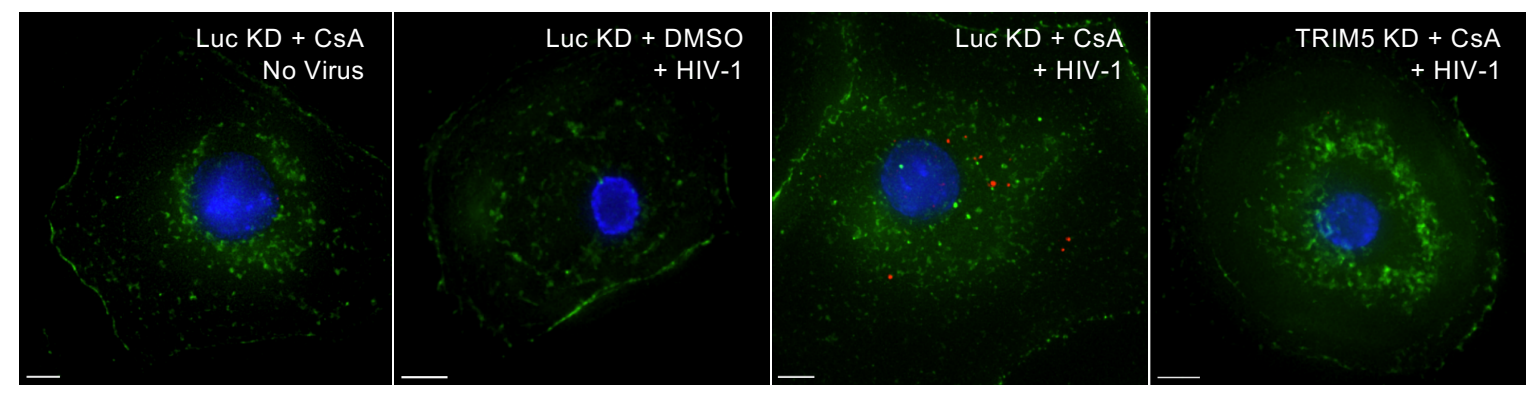

b

\section{Donor 1}

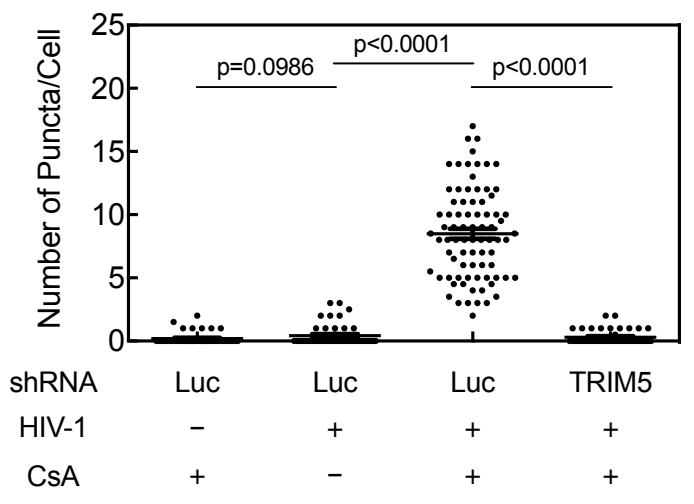

Donor 2

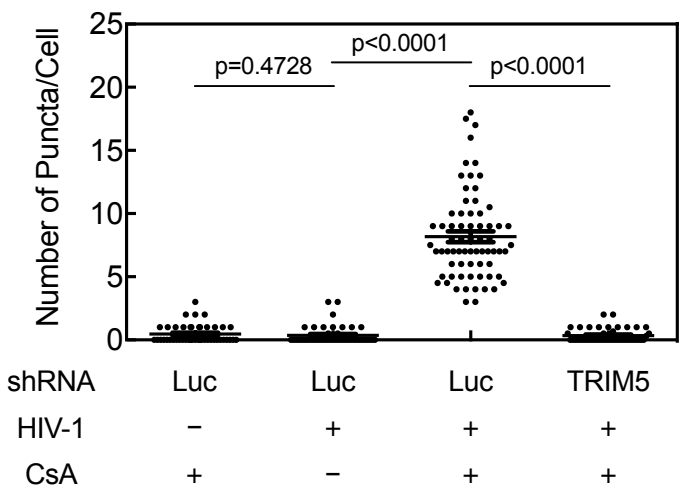

Fig. 4. Endogenous TRIM5a in primary human macrophages associates with HIV$1 \mathrm{CA}$ after acute challenge but only when the CA-CypA interaction is disrupted. a, Macrophages from two blood donors were transduced and selected with vector bearing shRNA targeting either TRIM5 or Luc. Cells were then challenged for 2 hrs with VSV G-

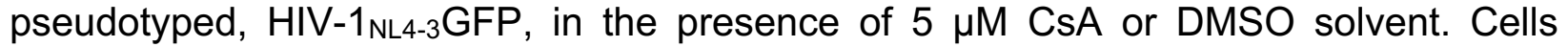
were fixed and the PLA assay was performed with antibodies against HIV-1 CA and TRIM5a. Representative images show PLA puncta (red), nuclei stained with Hoechst (blue), and actin filaments stained with phalloidin (green). $\mathbf{b}$. The number of puncta per cell in the PLA assay, after analysis of at least 45 cells, per condition, in triplicate (mean \pm SEM). Significance was determined by two-tailed, unpaired t-test. 


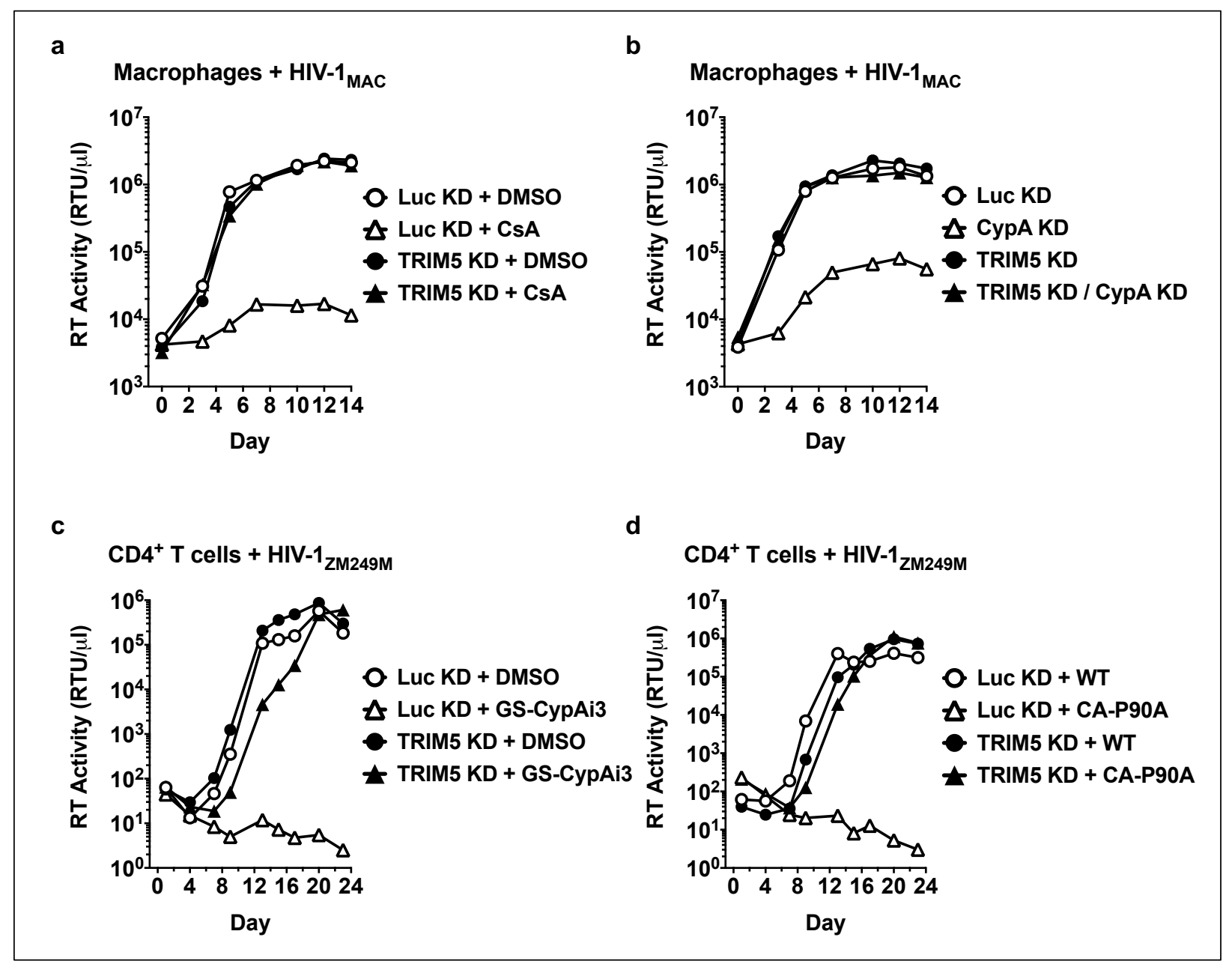

Fig. 5. Endogenous TRIM5a suppresses spreading infection of HIV-1 in human primary macrophages and $\mathrm{CD4}^{+}{ }^{+} \mathrm{T}$ cells when the CA-CypA interaction is

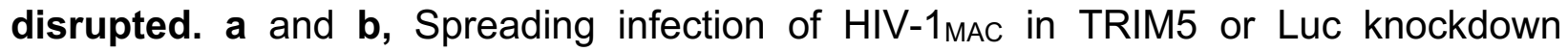
macrophages with $5 \mu \mathrm{M}$ CsA (a) or with vectors bearing shRNAs targeting CypA or Luc (b), as indicated. c and d, Spreading infection of HIV-1 Zm249M in CD4 ${ }^{+}$T cells expressing shRNA targeting TRIM5 or Luc with $2.5 \mu \mathrm{M}$ GS-CypAi3 (c) or when challenged with virus bearing CA-P90A (d), as indicated. HIV-1 replication was monitored by measuring reverse transcriptase (RT) activity in the culture supernatant over time. Data shown are representative of experiments in two blood donor cells for each condition. 